\title{
Chi-Square Mixture Representations for the Distribution of the Scalar Schur Complement in a Noncentral Wishart Matrix
}

\author{
Constantin Siriteanu ${ }^{\mathrm{a}}$, Satoshi Kuriki ${ }^{\mathrm{b}}$, Donald Richards ${ }^{\mathrm{c}}$, Akimichi Takemura ${ }^{\mathrm{d}}$ \\ ${ }^{a}$ Graduate School of Information Science and Technology, Osaka University, Osaka, Japan \\ ${ }^{b}$ The Institute of Statistical Mathematics, Tokyo, Japan \\ ${ }^{c}$ Department of Statistics, Pennsylvania State University, University Park, Pennsylvania, USA \\ ${ }^{d}$ Graduate School of Information Science and Technology, University of Tokyo, Tokyo, Japan
}

\begin{abstract}
We show that the distribution of the scalar Schur complement in a noncentral Wishart matrix is a mixture of central chi-square distributions with different degrees of freedom. For the case of a rank-1 noncentrality matrix, the weights of the mixture representation arise from a noncentral beta mixture of Poisson distributions.

Keywords: Confluent Appell function; noncentral beta distribution.
\end{abstract}

\section{Introduction}

\subsection{Motivating the study of the Schur complement}

This paper considers the distribution of the Schur complement in a real-valued noncentral Wishart matrix. The matrix Schur complement arises naturally in many areas of multivariate statistical analysis as the covariance matrix of the conditional Gaussian distribution, in decompositions of the Wishart matrix, and in hypothesis testing problems [1], [5], [22, Ch. 6]. In particular, the Schur complement is a crucial quantity in multivariate statistical inference with monotone incomplete data [3], [4].

The Schur complement also appears in multiple-input/multiple-output (MIMO) wireless communications systems, as follows. For a communications link over a randomly changing radio channel, the signal-to-noise ratio is a crucial metric that determines all other performance measures, e.g., error probability, outage probability, capacity. The signal-to-noise ratio is proportional with the scalar Schur complement in the sample correlation matrix formed with the random MIMO channel matrix when the received data is estimated by the least-squares-like method known as zero-forcing detection [18], [20], [19]. Knowing the distribution of the signal-to-noise ratio allows us to characterize the performance measures statistically, e.g., in average.

Email addresses: constantin. siriteanu@ist. osaka-u.ac.jp (Constantin Siriteanu), kuriki@ism.ac.jp (Satoshi Kuriki), richards@stat.psu.edu (Donald Richards), takemura@stat.t.u-tokyo.ac.jp (Akimichi Takemura) 
In the case of MIMO channel matrices that are complex-valued Gaussian distributed, we have derived recently analyses of performance measures by means of the Schur complement. For instance, in [18], [19] we characterized with infinite series the distribution of the scalar Schur complement in a complex-valued Wishart matrix with rank-1 noncentrality matrix. Furthermore, in [20], we characterized the more general matrix Schur complement, under certain conditions. However, the weights in those infinite series [18, Eq. (39)], [19, Eq. (59)] are alternately positive and negative, which can lead to numerical instability in evaluating ensuing MIMO performance measures [18, Sec. V.F], and that phenomenon partly motivated us to research mixture representations for the distribution of the scalar Schur complement, which we now do in this paper.

\subsection{Contributions and Organization of the Paper}

In this paper we consider the case of real-valued Gaussian distributed matrix with rank-1 mean, which yields a real-valued Wishart matrix with rank-1 noncentrality matrix. We first show that the distribution of the scalar Schur complement in this Wishart matrix is a mixture of noncentral $\chi^{2}$ distributions, where the noncentrality parameter is random. Since the noncentral $\chi^{2}$ distribution itself is a Poisson mixture of central $\chi^{2}$ distributions with different degrees of freedom, the distribution of the scalar Schur complement is also a mixture of central $\chi^{2}$ distributions with different degrees of freedom.

We also express the mixture weights in terms of known discrete probability distributions over the set of nonnegative integers. Whereas, in general, these weights are difficult to evaluate, for the case of a rank-1 noncentrality matrix, we prove that the weights are the probabilities arising from a noncentral beta mixture of Poisson distributions.

Thus, we prove in Section 2 that the distribution of the scalar Schur complement is a mixture of noncentral $\chi^{2}$ distributions, hence also a mixture of central $\chi^{2}$ distributions with different degrees of freedom. In Section 3 we relate the moment-generating function of the scalar Schur complement to the probability-generating function of the mixture weights of central $\chi^{2}$ distributions. In Section 4 we derive the distribution of the scalar Schur complement in a noncentral Wishart matrix with a rank-1 noncentrality matrix.

\subsection{Notation}

- Scalars, vectors, and matrices are represented with lowercase italics, lowercase boldface, and uppercase boldface, respectively, e.g., $u, \mathbf{x}$, and $\mathbf{X} ; \mathbb{R}^{n}$ and $\mathbb{R}^{n \times p}$ denote the set of $n$-dimensional column vectors and the set of $n \times p$ matrices, respectively; The zero vectors and matrices of appropriate dimensions are denoted by $\mathbf{0}$; the superscript $\therefore$ stands for transpose; $\mathbf{I}_{n}$ denotes the $n \times n$ identity matrix.

- The notation $\mathbf{x} \sim \mathcal{N}_{n}(\mathbf{m}, \boldsymbol{\Sigma})$ indicates an $n$-dimensional, real-valued, Gaussian random vector with mean $\mathbf{m}$ and covariance $\Sigma ; \mathbf{W} \sim \mathcal{W}_{p}(n, \boldsymbol{\Sigma}, \Omega)$ indicates that $\mathbf{W}$ has a $p \times p$ 
noncentral Wishart distribution with $n$ degrees of freedom, covariance matrix $\boldsymbol{\Sigma}$, and noncentrality matrix $\boldsymbol{\Omega}$ [9, Eq. (67)]; $\mathbf{x}_{1} \mid \mathbf{X}_{2}$ stands for $\mathbf{x}_{1}$ conditioned on $\mathbf{X}_{2} ; \mathbb{E}[\cdot]$ denotes expectation; $\chi_{v}^{2}(\delta)$ denotes the noncentral chi-square distribution with $v$ degrees of freedom and noncentrality parameter $\delta ; \chi_{v}^{2}$ denotes the central chi-square distribution with $v$ degrees of freedom; $\operatorname{Beta}(k, l)$ represents the central beta distribution with shape parameters $k$ and $l ; \operatorname{Beta}(k, l, \delta)$ represents the noncentral beta distribution with shape parameters $k$ and $l$, and noncentrality $\delta$; finally, $\operatorname{Pois}(\lambda)$ represents the Poisson distribution with mean $\lambda$.

- For $\operatorname{Re}(k), \operatorname{Re}(l)>0, \Gamma(k)=\int_{0}^{\infty} t^{k-1} e^{-t} \mathrm{~d} t$ denotes the gamma function and $B(k, l)=$ $\int_{0}^{1} t^{k-1}(1-t)^{l-1} \mathrm{~d} t$ denotes the beta function; $(x)_{n}$ is the rising factorial, where $(x)_{0}=1$ and $(x)_{n}=x(x+1) \cdots(x+n-1), n \geq 1 ;{ }_{1} F_{1}(\cdot ; \cdot ; \cdot)$ is the confluent hypergeometric function [16, Eq. (13.2.2), p. 322]:

$$
{ }_{1} F_{1}(b ; c ; w)=\sum_{l=0}^{\infty} \frac{(b)_{l}}{(c)_{l}} \frac{w^{l}}{l !} ;
$$

and $\Phi_{2}(\cdot, \cdot ; \cdot ;, \cdot)$ is the confluent Appell function [2, Eq. (1.3)]:

$$
\Phi_{2}\left(b, b^{\prime} ; c ; w, z\right)=\sum_{l=0}^{\infty} \sum_{m=0}^{\infty} \frac{(b)_{l}\left(b^{\prime}\right)_{m}}{(c)_{l+m}} \frac{w^{l} z^{m}}{l ! m !} .
$$

- Acronyms and abbreviations: p.d.f. for the probability density function, p.g.f. for the probability generating function, m.g.f. for the moment-generating function.

\section{Distribution of the scalar Schur complement in a noncentral Wishart matrix}

\subsection{The scalar Schur complement in a noncentral Wishart matrix}

Let $\mathbf{X}=\left(x_{i j}\right) \in \mathbb{R}^{n \times p}$ be a real-valued Gaussian random matrix with mean $\mathbf{M}=\left(\mu_{i j}\right) \in$ $\mathbb{R}^{n \times p}$, and whose rows are mutually independent $p$-dimensional vectors with covariance ma$\operatorname{trix} \boldsymbol{\Sigma}=\left(\sigma_{i j}\right) \in \mathbb{R}^{p \times p}$.

Let us partition $\mathbf{X}$ and $\mathbf{M}$ into the first column and the rest of columns, i.e.,

$$
\mathbf{X}=\left(\begin{array}{ll}
\mathbf{x}_{1} & \mathbf{X}_{2}
\end{array}\right), \quad \mathbf{M}=\left(\begin{array}{ll}
\mathbf{m}_{1} & \mathbf{M}_{2}
\end{array}\right),
$$

with $\mathbf{x}_{1}, \mathbf{m}_{1} \in \mathbb{R}^{n}$, and $\mathbf{X}_{2}, \mathbf{M}_{2} \in \mathbb{R}^{n \times(p-1)}$. Accordingly, we partition $\boldsymbol{\Sigma}$ as

$$
\boldsymbol{\Sigma}=\left(\begin{array}{ll}
\sigma_{11} & \boldsymbol{\sigma}_{21}^{\prime} \\
\sigma_{21} & \Sigma_{22}
\end{array}\right)
$$

Then, the conditional distribution of $\mathbf{x}_{1}$ given $\mathbf{X}_{2}$ is [15, p. 35]

$$
\mathbf{x}_{1} \mid \mathbf{X}_{2} \sim \mathcal{N}_{n}\left(\mathbf{m}_{1}+\left(\mathbf{X}_{2}-\mathbf{M}_{2}\right) \boldsymbol{\Sigma}_{22}^{-1} \boldsymbol{\sigma}_{21}, \sigma_{11 \cdot 2} \mathbf{I}_{n}\right),
$$


where

$$
\sigma_{11 \cdot 2}=\sigma_{11}-\boldsymbol{\sigma}_{21}^{\prime} \boldsymbol{\Sigma}_{22}^{-1} \boldsymbol{\sigma}_{21}
$$

is the variance of each element of $\mathbf{x}_{1} \mid \mathbf{X}_{2}$, and, by definition, also the Schur complement of $\boldsymbol{\Sigma}_{22}$ in $\boldsymbol{\Sigma}$.

Now, let us consider the matrix of interest,

$$
\mathbf{W}=\mathbf{X}^{\prime} \mathbf{X} \in \mathbb{R}^{p \times p} .
$$

It is well known that for $\mathbf{M} \neq \mathbf{0}$ we have $\mathbf{W} \sim \mathcal{W}_{p}(n, \boldsymbol{\Sigma}, \boldsymbol{\Omega})$ with $\boldsymbol{\Omega}=\boldsymbol{\Sigma}^{-1} \mathbf{M}^{\prime} \mathbf{M}$. If we partition $\mathbf{W}$ analogously to (2) as

$$
\mathbf{W}=\left(\begin{array}{ll}
w_{11} & \mathbf{w}_{21}^{\prime} \\
\mathbf{w}_{21} & \mathbf{W}_{22}
\end{array}\right)=\left(\begin{array}{ll}
\mathbf{x}_{1}^{\prime} \mathbf{x}_{1} & \mathbf{x}_{1}^{\prime} \mathbf{X}_{2} \\
\mathbf{X}_{2}^{\prime} \mathbf{x}_{1} & \mathbf{X}_{2}^{\prime} \mathbf{X}_{2}
\end{array}\right)
$$

then, by definition, the Schur complement of $\mathbf{W}_{22}$ in $\mathbf{W}$, which is the scalar Schur complement of interest here, is given by

$$
\begin{aligned}
w_{11 \cdot 2} & =w_{11}-\mathbf{w}_{21}^{\prime} \mathbf{W}_{22} \mathbf{W}_{21} \\
& =\mathbf{x}_{1}^{\prime} \mathbf{x}_{1}-\mathbf{x}_{1}^{\prime} \mathbf{X}_{2}\left(\mathbf{X}_{2}^{\prime} \mathbf{X}_{2}\right)^{-1} \mathbf{X}_{2}^{\prime} \mathbf{x}_{1} \\
& =\mathbf{x}_{1}^{\prime}\left(\mathbf{I}_{n}-\mathbf{X}_{2}\left(\mathbf{X}_{2}^{\prime} \mathbf{X}_{2}\right)^{-1} \mathbf{X}_{2}^{\prime}\right) \mathbf{x}_{1} \\
& =\mathbf{x}_{1}^{\prime} \mathbf{Q}_{2} \mathbf{x}_{1},
\end{aligned}
$$

where

$$
\mathbf{Q}_{2}=\mathbf{I}_{n}-\mathbf{X}_{2}\left(\mathbf{X}_{2}^{\prime} \mathbf{X}_{2}\right)^{-1} \mathbf{X}_{2}^{\prime}
$$

\subsection{Distribution of the conditioned scalar Schur complement}

Based on $\mathbf{x}_{1} \mid \mathbf{X}_{2}$ characterized in (1) we have

$$
\tilde{\mathbf{x}}_{1}=\mathbf{x}_{1}-\mathbf{X}_{2} \boldsymbol{\Sigma}_{22}^{-1} \boldsymbol{\sigma}_{21} \sim \mathcal{N}_{n}\left(\tilde{\mathbf{m}}_{1}, \sigma_{11 \cdot 2} \mathbf{I}_{n}\right)
$$

where

$$
\tilde{\mathbf{m}}_{1}=\mathbf{m}_{1}-\mathbf{M}_{2} \Sigma_{22}^{-1} \boldsymbol{\sigma}_{21} .
$$

Note that $\tilde{\mathbf{x}}_{1}$ is independent of $\mathbf{X}_{2}$, and that the distribution of $\mathbf{x}_{1} \mid \mathbf{X}_{2}$ is the distribution of $\tilde{\mathbf{x}}_{1}+\mathbf{X}_{2} \boldsymbol{\Sigma}_{22}^{-1} \boldsymbol{\sigma}_{21}$. By substituting $\mathbf{x}_{1}$ from (4) into (3) and using $\mathbf{X}_{2}^{\prime}\left(\mathbf{I}_{n}-\mathbf{X}_{2}\left(\mathbf{X}_{2}^{\prime} \mathbf{X}_{2}\right)^{-1} \mathbf{X}_{2}^{\prime}\right) \mathbf{X}_{2}=\mathbf{0}$, we can write the Schur complement of interest as

$$
w_{11 \cdot 2}=\tilde{\mathbf{x}}_{1}^{\prime}\left(\mathbf{I}_{n}-\mathbf{X}_{2}\left(\mathbf{X}_{2}^{\prime} \mathbf{X}_{2}\right)^{-1} \mathbf{X}_{2}^{\prime}\right) \tilde{\mathbf{x}}_{1} .
$$


Now, let us define:

$$
\begin{aligned}
& \rho=\frac{w_{11 \cdot 2}}{\sigma_{11 \cdot 2}}=\frac{\tilde{\mathbf{x}}_{1}^{\prime} \mathbf{Q}_{2} \tilde{\mathbf{x}}_{1}}{\sigma_{11 \cdot 2}}, \\
& u=\frac{\tilde{\mathbf{m}}_{1}^{\prime} \mathbf{Q}_{2} \tilde{\mathbf{m}}_{1}}{\left\|\tilde{\mathbf{m}}_{1}\right\|^{2}} \\
& \lambda=\frac{\left\|\tilde{\mathbf{m}}_{1}\right\|^{2}}{\sigma_{11 \cdot 2}}, \\
& \delta=\lambda u=\frac{\tilde{\mathbf{m}}_{1}^{\prime} \mathbf{Q}_{2} \tilde{\mathbf{m}}_{1}}{\sigma_{11 \cdot 2}} .
\end{aligned}
$$

Because the $n \times n$ matrix $\mathbf{Q}_{2}$ is idempotent, i.e., the magnitude of its eigenvalues is either 0 or 1 , we deduce that $u \in[0,1]$. Furthermore, the fact that $\mathbf{Q}_{2}$ has $p-1$ eigenvalues equal to 0 and $v=n-p+1$ eigenvalues equal to 1 , yields, in general, the following noncentral $\chi^{2}$ distribution for the scaled Schur complement $\rho$ conditioned on $u$ [14]:

$$
\rho \mid u \sim \chi_{v}^{2}(\delta), \quad v=n-p+1 .
$$

Thus, the p.d.f. of $\rho \mid u$ is given by [14, p. 27] as

$$
g(w ; v, \delta)=\sum_{k=0}^{\infty} \frac{(\delta / 2)^{k}}{k !} e^{-\delta / 2} g_{v+2 k}(w), w>0,
$$

where

$$
g_{m}(w)=\frac{1}{2^{m / 2} \Gamma(m / 2)} w^{m / 2-1} e^{-w / 2}
$$

is the p.d.f. of a $\chi_{m}^{2}$ random variable.

\section{Relationship between the mixture m.g.f. and the mixture-weights p.g.f.}

\subsection{M.g.f.-p.g.f. relationship}

Note first that the distribution of $\rho \mid u$, characterized by the p.d.f. $g(w ; v, \delta)$ in (9), is a mixture of central $\chi^{2}$ distributions. Note also that the mixture weight, i.e.,

$$
\alpha_{k}=\frac{(\delta / 2)^{k}}{k !} e^{-\delta / 2}
$$

represents the probability that the Poisson random variable $A \sim \operatorname{Pois}(\delta / 2)$ takes the value $k$. On the one hand, the p.g.f. of $A$ is given by

$$
G_{A}(s)=\mathbb{E}\left[s^{A}\right]=\sum_{k=0}^{\infty} s^{k} \alpha_{k}=e^{(\delta / 2)(s-1)} .
$$

On the other hand, the m.g.f. of $\rho \mid u$ is given by the expression

$$
M_{\rho \mid u}(\theta)=\mathbb{E}\left[e^{\theta \rho} \mid u\right]=(1-2 \theta)^{-v / 2} \exp \left(\frac{\delta \theta}{1-2 \theta}\right),
$$

which is simpler than the corresponding p.d.f. expression in (9). 
Remark 1. For the conditioned Schur complement $\rho \mid u$, the m.g.f. from (12) of the mixture distribution in (9) and the p.g.f. from (11) of the mixture weights in (10) are related as

$$
M_{\rho \mid u}(\theta)=(1-2 \theta)^{-v / 2} G_{A}\left(\frac{1}{1-2 \theta}\right) .
$$

This reveals the equivalence between expressing the m.g.f. of a mixture of central $\chi^{2}$ distributions and expressing the p.g.f. of the discrete random variable characterized by the mixture weights. Hereafter, we refer to the p.g.f. of the discrete random variable as the p.g.f. of the mixture weights.

\subsection{General characterization of the unconditional distribution of the Schur complement}

Recall now from (7) that $\delta=\lambda u$, with $u$ being the random quadratic form defined in (5). Thus, we need to average the above distribution of $\rho \mid u$ over the distribution of $u$. Letting the p.d.f. of $u$ be denoted by $h(u)$, which is nonzero only for $u \in[0,1]$, the p.d.f. and m.g.f. of the scaled scalar Schur complement are given, respectively, by

$$
\begin{aligned}
p_{\rho}(w) & =\int_{0}^{1} g(w ; v, \lambda u) h(u) \mathrm{d} u \\
& =\sum_{k=0}^{\infty} g_{v+2 k}(w) \frac{(\lambda / 2)^{k}}{k !} \int_{0}^{1} u^{k} e^{-\lambda u / 2} h(u) \mathrm{d} u,
\end{aligned}
$$

and

$$
M_{\rho}(\theta)=(1-2 \theta)^{-v / 2} \int_{0}^{1} \exp \left(\frac{\lambda \theta u}{1-2 \theta}\right) h(u) \mathrm{d} u .
$$

Thus, the study of the distribution of $\rho$ reduces to the study of the density $h(u)$ of $u$, which, in general, is difficult to derive. However, regardless of $h(u)$, it is straightforward to show that an m.g.f.-p.g.f. relationship analogous to (13) holds also after removing the conditioning on $u$. One can show this directly by averaging over $u$ in (13).

Nonetheless, for more details, let us arrive at the same result by casting the p.d.f. from (14) as the mixture of central $\chi^{2}$ p.d.f.s

$$
p_{\rho}(w)=\sum_{k=0}^{\infty} \beta_{k} g_{v+2 k}(w)
$$

with mixture weights

$$
\beta_{k}=\frac{(\lambda / 2)^{k}}{k !} \int_{0}^{1} u^{k} e^{-\lambda u / 2} h(u) \mathrm{d} u, \quad k=0,1, \ldots
$$


We may view each weight $\beta_{k}$ as the probability that a nonnegative discrete random variable $B$ takes value $k$ because

$$
\begin{aligned}
\sum_{k=0}^{\infty} \beta_{k} & =\sum_{k=0}^{\infty} \beta_{k} \int_{0}^{\infty} g_{v+2 k}(w) \mathrm{d} w \\
& =\int_{0}^{\infty} \sum_{k=0}^{\infty} \beta_{k} g_{v+2 k}(w) \mathrm{d} w=\int_{0}^{\infty} p_{\rho}(w) \mathrm{d} w=1,
\end{aligned}
$$

where the interchange of summation and integration can be justified by Fubini's theorem. Let $G_{B}(s)$ be the p.g.f. of this random variable.

On the other hand, the m.g.f. corresponding to the p.d.f. in (16) can be written as

$$
\begin{aligned}
M_{\rho}(\theta) & =\int_{0}^{\infty} e^{\theta w} \sum_{k=0}^{\infty} \beta_{k} g_{v+2 k}(w) \mathrm{d} w \\
& =\sum_{k=0}^{\infty} \beta_{k} \int_{0}^{\infty} e^{\theta w} g_{v+2 k}(w) \mathrm{d} w \\
& =(1-2 \theta)^{-v / 2} \sum_{k=0}^{\infty}(1-2 \theta)^{-k} \beta_{k} .
\end{aligned}
$$

Proposition 1. For the Schur complement $\rho$, regardless of $h(u)$, the mixture-distribution m.g.f. from (18) and the p.g.f. of the corresponding mixture weights in (17) are related as follows:

$$
M_{\rho}(\theta)=(1-2 \theta)^{-v / 2} G_{B}\left(\frac{1}{1-2 \theta}\right) .
$$

The usefulness of this equivalence is supported below, as we characterize the distribution of $\rho$ for the case with $\operatorname{rank} \boldsymbol{\Omega}=1$.

\section{Distribution of the Schur complement with $\tilde{\mathrm{m}}_{1} \neq 0$ and $\operatorname{rank} M=\operatorname{rank} \Omega=1$}

The trivial case with $\tilde{\mathbf{m}}_{1}=\mathbf{0}$, i.e., with $\mathbf{m}_{1}=\mathbf{M}_{2} \boldsymbol{\Sigma}_{22}^{-1} \boldsymbol{\sigma}_{21}$, implies that $u=0$, which, based on (8), yields, simply, $\rho=\rho \mid u \sim \chi_{v}^{2}(\delta), v=n-p+1$, [19]. Therefore, hereafter, we consider the case with $\tilde{\mathbf{m}}_{1} \neq \mathbf{0}$ and, for tractability, $\operatorname{rank} \boldsymbol{\Omega}=1$. Then, results shown above help recast more conveniently results deduced for $\mathbf{M}_{2}=\mathbf{0}$ and $\mathbf{M}_{2} \neq \mathbf{0}$ in [18] and [19], respectively.

\subsection{Case with $\mathbf{m}_{1} \neq \mathbf{0}$ and $\mathbf{M}_{2}=\mathbf{0}$, i.e., $\operatorname{rank} \mathbf{M}=\operatorname{rank} \boldsymbol{\Omega}=1$}

Let the QR decomposition of $\mathbf{X}_{2} \in \mathbb{R}^{n \times(p-1)}$ be

$$
\mathbf{X}_{2}=\mathbf{H T}
$$


where $\mathbf{T} \in \mathbb{R}^{(p-1) \times(p-1)}$ is upper triangular with positive diagonal elements, and $\mathbf{H} \in \mathbb{R}^{n \times(p-1)}$ satisfies $\mathbf{H}^{\prime} \mathbf{H}=\mathbf{I}_{p-1}$. Thus, for $\mathbf{M}_{2}=\mathbf{0}, \mathbf{H}$ has the uniform distribution over the Stiefel manifold $\left\{\mathbf{H} \mid \mathbf{H}^{\prime} \mathbf{H}=\mathbf{I}_{p-1}\right\}$. Then, we can write for $\mathbf{Q}_{2}$ the following

$$
\mathbf{Q}_{2}=\mathbf{I}_{n}-\mathbf{X}_{2}\left(\mathbf{X}_{2}^{\prime} \mathbf{X}_{2}\right)^{-1} \mathbf{X}_{2}^{\prime}=\mathbf{I}_{n}-\mathbf{H} \mathbf{H}^{\prime}
$$

and for $u$ the following

$$
u=\frac{\tilde{\mathbf{m}}_{1}^{\prime} \mathbf{Q}_{2} \tilde{\mathbf{m}}_{1}}{\left\|\tilde{\mathbf{m}}_{1}\right\|^{2}}=\frac{\tilde{\mathbf{m}}_{1}^{\prime}}{\left\|\tilde{\mathbf{m}}_{1}\right\|}\left(\mathbf{I}_{n}-\mathbf{H H}^{\prime}\right) \frac{\tilde{\mathbf{m}}_{1}}{\left\|\tilde{\mathbf{m}}_{1}\right\|} \sim \operatorname{Beta}(v / 2,(p-1) / 2) .
$$

Thus, $u$ has the central beta p.d.f.

$$
h(u)=\frac{u^{v / 2-1}(1-u)^{(p-1) / 2-1}}{B(v / 2,(p-1) / 2)} .
$$

With the p.d.f. $h(u)$ from (20), the m.g.f. of interest, i.e., $M_{\rho}(\theta)$ with $\rho=\lambda u$ from (15), can be written, based on [16, Eq. (13.4.1)], as

$$
\begin{aligned}
M_{\rho}(\theta) & =(1-2 \theta)^{-v / 2} \int_{0}^{1} \exp \left(\frac{\lambda \theta u}{1-2 \theta}\right) h(u) \mathrm{d} u \\
& =(1-2 \theta)^{-v / 2}{ }_{1} F_{1}\left(\frac{v}{2} ; \frac{n}{2} ; \frac{\lambda \theta}{1-2 \theta}\right) .
\end{aligned}
$$

On the other hand, the positive weight for the corresponding mixture distribution characterized by (16), i.e., $\beta_{k}$ defined in (17), is given by [16, Eq. (13.4.1)]

$$
\begin{aligned}
\beta_{k} & =\int_{0}^{1} \frac{u^{v / 2-1}(1-u)^{(p-1) / 2-1}}{B(v / 2+k,(p-1) / 2)} \frac{(\lambda u / 2)^{k}}{k !} e^{-\lambda u / 2} \mathrm{~d} u \\
& =\frac{(\lambda / 2)^{k}}{k !} \frac{(\nu / 2)_{k}}{(n / 2)_{k}} F_{1}\left(\frac{v}{2}+k ; \frac{n}{2}+k ;-\frac{\lambda}{2}\right) .
\end{aligned}
$$

Remark 2. Note from (22) that the distribution of random variable B with $\operatorname{Pr}(B=k)=\beta_{k}$ is a beta mixture of Poisson distributions [6, p. 42], [7], [21], which, in turn, is a particular case of the generalized hypergeometric factorial moment distribution [10, Secs. 2.4.2, 6.1.13], [12], [13].

Because the integral and infinite series expressions deduced above for $\beta_{k}$ are involved, it is not trivial to deduce for $\beta_{k}$ the corresponding p.g.f. $G_{B}(s)=\mathbb{E}\left[s^{B}\right]=\sum_{k=0}^{\infty} s^{k} \beta_{k}$. On the other hand, by applying the general relationship revealed in (19) for the m.g.f. in (21), the p.g.f. for mixture weights $\beta_{k}$ can be expressed — surprisingly simply — as

$$
G_{B}(s)={ }_{1} F_{1}\left(\frac{\nu}{2} ; \frac{n}{2} ; \frac{\lambda}{2}(s-1)\right) .
$$




\subsection{Case with $\mathbf{m}_{1} \neq \mathbf{0}$ and $\mathbf{M}_{2} \neq \mathbf{0}$, but $\operatorname{rank} \mathbf{M}=\operatorname{rank} \boldsymbol{\Omega}=1$}

Recall from above that for $\mathbf{m}_{1} \neq \mathbf{0}$ and $\mathbf{M}_{2}=\mathbf{0}, u$ was shown to be central beta distributed. On the other hand, when $\mathbf{m}_{1} \neq \mathbf{0}$ and $\mathbf{M}_{2} \neq \mathbf{0}$, and, further, $\operatorname{rank} \mathbf{M}=\operatorname{rank} \boldsymbol{\Omega}=1$, we reveal next that $u$ is noncentral beta distributed (cf. [8], [11, Section 30.7.2]), [17].

Proposition 2. When $\mathbf{m}_{1} \neq \mathbf{0}, \mathbf{M}_{2} \neq \mathbf{0}$, and $\operatorname{rank} \mathbf{M}=\operatorname{rank} \boldsymbol{\Omega}=1$, random variable $u$ has the distribution Beta $(v / 2,(p-1) / 2, \tau)$ with $\tau=\operatorname{tr}\left[\Sigma_{22}^{-1} \mathbf{M}_{2}^{\prime} \mathbf{M}_{2}\right]$, i.e., $u$ has the p.d.f.

$$
h(u)=e^{-\tau / 2} \sum_{l=0}^{\infty} \frac{(\tau / 2)^{l}}{l !} \frac{u^{v / 2-1}(1-u)^{(p-1) / 2+l-1}}{B(v / 2,(p-1) / 2+l)} .
$$

Then, the m.g.f. of the scaled scalar Schur complement, the corresponding mixture weights, and their p.g.f. can be expressed as follows:

$$
\begin{gathered}
M_{\rho}(\theta)=(1-2 \theta)^{-v / 2} e^{-\tau / 2} \sum_{l=0}^{\infty} \frac{(\tau / 2)^{l}}{l !}{ }_{1} F_{1}\left(\frac{v}{2} ; \frac{n}{2}+l ; \frac{\lambda \theta}{1-2 \theta}\right) \\
=(1-2 \theta)^{-v / 2} e^{-\tau / 2} \Phi_{2}\left(\frac{v}{2}, \frac{n}{2} ; \frac{n}{2} ; \frac{\lambda \theta}{1-2 \theta}, \frac{\tau}{2}\right), \\
\beta_{k}=\frac{(\lambda / 2)^{k}}{k !} \sum_{l=0}^{\infty} \frac{(\tau / 2)^{l}}{l !} \frac{(v / 2)_{k}}{(n / 2+l)_{k}}{ }_{1} F_{1}\left(\frac{v}{2}+k ; \frac{n}{2}+k+l ;-\frac{\lambda}{2}\right) \\
=\frac{(\lambda / 2)^{k}}{k !} \frac{(v / 2)_{k}}{(n / 2)_{k}} \Phi_{2}\left(\frac{v}{2}+k, \frac{n}{2} ; \frac{n}{2}+k ;-\frac{\lambda}{2}, \frac{\tau}{2}\right), \\
G(s)=e^{-\tau / 2} \sum_{l=0}^{\infty} \frac{(\tau / 2)^{l}}{l !}{ }_{1} F_{1}\left(\frac{v}{2} ; \frac{n}{2}+l ; \frac{\lambda}{2}(s-1)\right) \\
=e^{-\tau / 2} \Phi_{2}\left(\frac{v}{2}, \frac{n}{2} ; \frac{n}{2} ; \frac{\lambda}{2}(s-1), \frac{\tau}{2}\right) .
\end{gathered}
$$

We remark that, to the best of our knowledge, the p.g.f. in (26) has not been studied before now.

Proof. Let us provide for (23) a new proof, different from the one in [19], based on known distributional results on Hotelling's $T^{2}$-statistic (cf. [1, Ch. 5]).

First, let us transform the parameter matrices $\Sigma$ and $\mathbf{M}$ to simpler forms. This can be done by noting that $\tilde{\mathbf{x}}_{1}$ had been obtained in (4) by the following column-wise linear transformation of $\mathbf{X}$ :

$$
\tilde{\mathbf{x}}_{1}=\mathbf{x}_{1}-\mathbf{X}_{2} \Sigma_{22}^{-1} \sigma_{21}=\left(\begin{array}{ll}
\mathbf{x}_{1} & \mathbf{X}_{2}
\end{array}\right)\left(\begin{array}{c}
1 \\
-\Sigma_{22}^{-1} \sigma_{21}
\end{array}\right)=\mathbf{X}\left(\begin{array}{c}
1 \\
-\Sigma_{22}^{-1} \sigma_{21}
\end{array}\right)
$$


Thus, employing

$$
\mathbf{A}=\left(\begin{array}{cc}
1 & \mathbf{0}^{\prime} \\
-\boldsymbol{\Sigma}_{22}^{-1} \boldsymbol{\sigma}_{21} & \mathbf{I}_{p-1}
\end{array}\right)
$$

for the column-wise linear transformation $\mathbf{X} \mapsto \mathbf{X A}$ yields

$$
\mathbf{X} \mapsto \mathbf{X A}=\left(\begin{array}{ll}
\mathbf{x}_{1} & \mathbf{X}_{2}
\end{array}\right)\left(\begin{array}{cc}
1 & \mathbf{0}^{\prime} \\
-\boldsymbol{\Sigma}_{22}^{-1} \boldsymbol{\sigma}_{21} & \mathbf{I}_{p-1}
\end{array}\right)=\left(\begin{array}{ll}
\tilde{\mathbf{x}}_{1} & \mathbf{X}_{2}
\end{array}\right),
$$

which preserves the Schur complement $w_{11 \cdot 2}$ of $\mathbf{W}=\mathbf{X}^{\prime} \mathbf{X}$. On the other hand, this transformation induces the transformation on the parameter space of $\mathbf{M}$ and $\boldsymbol{\Sigma}$ as follows:

$$
\begin{aligned}
& \mathbf{M} \mapsto \mathbf{M A}=\left(\begin{array}{ll}
\tilde{\mathbf{m}}_{1} & \mathbf{M}_{2}
\end{array}\right), \\
& \boldsymbol{\Sigma} \mapsto \mathbf{A}^{\prime} \boldsymbol{\Sigma} \mathbf{A}=\left(\begin{array}{cc}
\sigma_{11 \cdot 2} & \mathbf{0}^{\prime} \\
\mathbf{0} & \boldsymbol{\Sigma}_{22}
\end{array}\right) .
\end{aligned}
$$

Note that this preserves $\operatorname{rank} \mathbf{M}=\operatorname{rank}(\mathbf{M A})=1$.

Second, let us apply the row-wise transformation $\mathbf{X} \mapsto \mathbf{P X}$, with orthogonal matrix $\mathbf{P} \in$ $\mathbb{R}^{n \times n}$ specified below. Then, $\mathbf{W}=\mathbf{X}^{\prime} \mathbf{X}$ and the covariance structure $\boldsymbol{\Sigma}$ of $\mathbf{X}$ are unchanged, whereas mean matrix is transformed as $\mathbf{M} \mapsto \mathbf{P M}$.

Since $\operatorname{rank}(\mathbf{M A})=1$, there exists an $n$-dimensional column vector a and a $p$-dimensional column vector $\mathbf{b}=\left(b_{1}, \ldots, b_{p}\right)^{\prime}$ such that

$$
\mathbf{M A}=\mathbf{a b}^{\prime},
$$

where we may assume $\|\mathbf{a}\|=1$, because $\mathbf{a}$ and $\mathbf{b}$ are nonzero vectors and $\mathbf{a} \mathbf{b}^{\prime}=(\mathbf{a} / c) c \mathbf{b}^{\prime}$ for any constant $c \neq 0$. Then, by (27) and (6) we have

$$
\mathbf{a}=\frac{\tilde{\mathbf{m}}_{1}}{\left\|\tilde{\mathbf{m}}_{1}\right\|}, \quad \text { and } \quad b_{1}=\left\|\tilde{\mathbf{m}}_{1}\right\|=\sqrt{\lambda \sigma_{11 \cdot 2}} .
$$

Constructing $\mathbf{P}$ so that its first row is $\mathbf{a}^{\prime}$ and its remaining rows are orthogonal to a yields

$$
\mathbf{P} \mathbf{M} \mathbf{A}=\mathbf{P} \mathbf{a} \mathbf{b}^{\prime}=\left(\begin{array}{c}
1 \\
0 \\
\vdots \\
0
\end{array}\right) \mathbf{b}^{\prime}=\left(\begin{array}{ccccc}
\sqrt{\lambda \sigma_{11 \cdot 2}} & b_{2} & b_{3} & \ldots & b_{p} \\
0 & 0 & 0 & \ldots & 0 \\
\vdots & \vdots & \vdots & & \vdots \\
0 & 0 & 0 & \ldots & 0
\end{array}\right) .
$$

The assumption $\mathbf{M}_{2} \neq \mathbf{0}$ implies that $\mathbf{b}_{2}=\left(b_{2}, \ldots, b_{p}\right)^{\prime} \neq \mathbf{0}$. Therefore, it suffices to prove the proposition when $\boldsymbol{\Sigma}$ and $\mathbf{M}$ are as follows:

$$
\boldsymbol{\Sigma}=\left(\begin{array}{cc}
\sigma_{11 \cdot 2} & \mathbf{0}^{\prime} \\
\mathbf{0} & \boldsymbol{\Sigma}_{22}
\end{array}\right), \quad \mathbf{M}=\left(\begin{array}{cccc}
\sqrt{\lambda \sigma_{11 \cdot 2}} & b_{2} & \cdots & b_{p} \\
0 & 0 & \cdots & 0 \\
\vdots & \vdots & & \vdots \\
0 & 0 & \cdots & 0
\end{array}\right) .
$$


Note that in the matrix in (28), in the first column, which corresponds to $\tilde{\mathbf{m}}_{1}$ from (1), only the first element is nonzero. Therefore, the random variable $u$ from $(5)$ is the $(1,1)$ element of the matrix $\mathbf{Q}_{2}=\mathbf{I}_{n}-\mathbf{X}_{2}\left(\mathbf{X}_{2}^{\prime} \mathbf{X}_{2}\right)^{-1} \mathbf{X}_{2}^{\prime}$. This element can be written, by partitioning $\mathbf{X}_{2}$ into its first row and its remaining rows as

$$
\mathbf{X}_{2}=\left(\begin{array}{l}
\mathbf{x}_{12}^{\prime} \\
\mathbf{X}_{22}
\end{array}\right)
$$

and by applying the matrix inversion lemma, as follows:

$$
\begin{aligned}
u & =1-\mathbf{x}_{12}^{\prime}\left(\mathbf{X}_{22}^{\prime} \mathbf{X}_{22}+\mathbf{x}_{12} \mathbf{x}_{12}^{\prime}\right)^{-1} \mathbf{x}_{12} \\
& =1-\mathbf{x}_{12}^{\prime}\left(\left(\mathbf{X}_{22}^{\prime} \mathbf{X}_{22}\right)^{-1}-\frac{\left(\mathbf{X}_{22}^{\prime} \mathbf{X}_{22}\right)^{-1} \mathbf{x}_{12} \mathbf{x}_{12}^{\prime}\left(\mathbf{X}_{22}^{\prime} \mathbf{X}_{22}\right)^{-1}}{1+\mathbf{x}_{12}^{\prime}\left(\mathbf{X}_{22}^{\prime} \mathbf{X}_{22}\right)^{-1} \mathbf{x}_{12}}\right) \mathbf{x}_{12} \\
& =1-\frac{\mathbf{x}_{12}\left(\mathbf{X}_{22}^{\prime} \mathbf{X}_{22}\right)^{-1} \mathbf{x}_{12}^{\prime}}{1+\mathbf{x}_{12}^{\prime}\left(\mathbf{X}_{22}^{\prime} \mathbf{X}_{22}\right)^{-1} \mathbf{x}_{12}}=\frac{1}{1+\mathbf{x}_{12}^{\prime}\left(\mathbf{X}_{22}^{\prime} \mathbf{X}_{22}\right)^{-1} \mathbf{x}_{12}}
\end{aligned}
$$

Here $\mathbf{x}_{12}^{\prime}$ and the rows of $\mathbf{X}_{22}$ are independent Gaussian vectors with means $\mathbf{b}_{2}$ and $\mathbf{0}$, respectively, but the same covariance matrix $\boldsymbol{\Sigma}_{22}$. Then, by [1, Theorems 5.2.2, 5.4.1], the scalar random variable $T^{2}=\mathbf{x}_{12}^{\prime}\left(\mathbf{X}_{22}^{\prime} \mathbf{X}_{22}\right)^{-1} \mathbf{x}_{12}$ is a statistic of Hotelling's $T^{2}$-type, and therefore has the noncentral $F$-distribution with p.d.f.

$$
f_{T^{2}}(t)=e^{-\tau / 2} \sum_{l=0}^{\infty} \frac{(\tau / 2)^{l}}{l !} \frac{1}{B(v / 2,(p-1) / 2+l)} \frac{t^{p / 2-1+l}}{(1+t)^{n / 2+l}},
$$

where $\tau$ is the trace of the noncentrality matrix for the Wishart distribution of $\mathbf{W}_{22}$, i.e.,

$$
\tau=\operatorname{tr}\left[\boldsymbol{\Sigma}_{22}^{-1} \mathbf{M}_{2}^{\prime} \mathbf{M}_{2}\right]=\operatorname{tr}\left[\mathbf{P} \mathbf{M}_{2} \boldsymbol{\Sigma}_{22}^{-1}\left(\mathbf{P} \mathbf{M}_{2}\right)^{\prime}\right]=\mathbf{b}_{2} \boldsymbol{\Sigma}_{22}^{-1} \mathbf{b}_{2}^{\prime}
$$

Then the transformation $u=1 /\left(1+T^{2}\right)$ yields for $u$ the p.d.f. from (23).

Finally, using this p.d.f. along with results from Section 3 produces (24), (25), and (26).

\section{Acknowledgments}

A. Takemura acknowledges the support of the JSPS Grant-in-Aid for Scientific Research No. 25220001.

\section{References}




\section{References}

[1] T. W. Anderson. An Introduction to Multivariate Statistical Analysis. WileyInterscience, Hoboken, NJ, third edition, 2003.

[2] Y. A. Brychkov and N. Saad. Some formulas for the Appell function $F_{1}\left(a, b, b^{\prime} ; c ; w, z\right)$. Integral Transforms Spec. Funct., 23(11):793-802, 2012.

[3] W.-Y. Chang and D. St. P. Richards. Finite-sample inference with monotone incomplete multivariate normal data. I. J. Multivariate Anal., 100(9):1883-1899, 2009.

[4] W.-Y. Chang and D. St. P. Richards. Finite-sample inference with monotone incomplete multivariate normal data. II. J. Multivariate Anal., 101(3):603-620, 2010.

[5] R. W. Cottle. Manifestations of the Schur complement. Linear Algebra Appl., 8(3):189211, 1974.

[6] J. Grandell. Mixed Poisson Processes. Chapman \& Hall, London, 1997.

[7] J. Gurland. A generalized class of contagious distributions. Biometrics, 14:229-249, 1958.

[8] J. L. Hodges, Jr. On the noncentral beta-distribution. Ann. Math. Statist., 26:648-653, 1955.

[9] A. T. James. Distributions of matrix variates and latent roots derived from normal samples. Ann. Math. Statist., 35:475-501, 1964.

[10] N. L. Johnson, A. W. Kemp, and S. Kotz. Univariate Discrete Distributions. WileyInterscience, Hoboken, NJ, third edition, 2005.

[11] N. L. Johnson, S. Kotz, and N. Balakrishnan. Continuous Univariate Distributions. Vol. 2. Wiley, New York, second edition, 1995.

[12] A. W. Kemp and C. D. Kemp. A family of discrete distributions defined via their factorial moments. Comm. Statist., 3:1187-1196, 1974.

[13] I.-L. Lu and D. St. P. Richards. Natural exponential families and generalized hypergeometric measures. Comm. Statist. Theory Methods, 37:2472-2487, 2008.

[14] A. M. Mathai, S. B. Provost, and T. Hakayawa. Bilinear Forms and Zonal Polynomials. Springer, New York, 1995.

[15] R. J. Muirhead. Aspects of Multivariate Statistical Theory. Wiley, New York, 1982. 
[16] F. W. J. Olver, D. W. Lozier, R. F. Boisvert, and C. W. Clarck, editors. NIST Handbook of Mathematical Functions. Cambridge University Press, New York, 2010.

[17] G. A. F. Seber. The non-central chi-squared and beta distributions. Biometrika, 50:542544, 1963.

[18] C. Siriteanu, S. D. Blostein, A. Takemura, H. Shin, S. Yousefi, and S. Kuriki. Exact MIMO zero-forcing detection analysis for transmit-correlated Rician fading. IEEE Trans. Wireless Commun., 13:1514-1527, 2014.

[19] C. Siriteanu, A. Takemura, C. Koutschan, S. Kuriki, D. Richards, and H. Shin. Exact $\mathrm{ZF}$ analysis and computer-algebra-aided evaluation in rank-1 LoS rician fading. IEEE Trans. Wireless Commun., submitted, July 2015.

[20] C. Siriteanu, A. Takemura, S. Kuriki, D. Richards, and H. Shin. Schur complement based analysis of MIMO zero-forcing for Rician fading. IEEE Trans. Wireless Commun., 14:1757-1771, 2015.

[21] P. F. M. Teunis and A. H. Havelaar. The beta Poisson dose-response model is not a single-hit model. Risk Analysis, 20:513-520, 2000.

[22] F. Zhang, editor. The Schur Complement and its Applications. Springer, New York, 2005. 